\title{
Efficacy of Intravenous Use of Lidocaine in Postoperative Pain Management After Laparoscopic Colorectal Surgery: A Meta-analysis and Meta-regression of RCTs
}

\author{
CHAMAIDI SARAKATSIANOU ${ }^{1}$, KONSTANTINOS PERIVOLIOTIS ${ }^{2}$, \\ GEORGE TZOVARAS ${ }^{2}$, ATHINA A. SAMARA ${ }^{2}$ and IOANNIS BALOYIANNIS ${ }^{2}$ \\ ${ }^{1}$ Department of Anesthesiology, University Hospital of Larissa, Larissa, Greece; \\ ${ }^{2}$ Department of Surgery, University Hospital of Larissa, Larissa, Greece
}

\begin{abstract}
Background/Aim: Current literature reports regarding the effect of lidocaine in laparoscopic colectomies are still inconclusive. The purpose of this study was to review the current literature and estimate the overall effect of intravenous lidocaine administration in postoperative recovery of patients submitted to laparoscopic colectomies. Materials and Methods: This study was completed based on the PRISMA guidelines and the Cochrane Handbook for Systematic Reviews of Interventions. A systematic screening using scholar databases was performed (Medline, Scopus, Web of Science, CENTRAL). Results: In total, 8 studies and 407 patients were included in this meta-analysis. Introduction of intravenous lidocaine in the perioperative analgesia scheme did not improve hospitalization duration $(p=0.23)$, morphine consumption $(p=0.96)$, perioperative bowel function (first flatus $p=0.40$, first bowel opened $p=0.13$, first diet $p=0.16$ ), or the overall complication rates $(p=0.42)$. Overall, high heterogeneity levels were identified. Conclusion: Current evidence indicates that lidocaine does not improve rehabilitation after laparoscopic colectomies.
\end{abstract}

The implementation of the enhanced recovery after surgery programs (ERAS) and the enhanced recovery protocols (ERP) has resulted in notable improvement of the postoperative outcomes in colorectal surgery (1-6). In order to reduce hospitalization duration and complication rates of abdominal surgery, these multidisciplinary protocols

This article is freely accessible online.

Correspondence to: Athina A. Samara (ORCID: 0000-0002-61777281), MD, MSc, Department of Surgery, University Hospital of Larissa, Mezourlo, 41110, Larissa, Greece. Tel: +30 2413501701, e-mail: at.samara93@gmail.com

Key Words: Lidocaine, laparoscopic, colorectal, meta-analysis. proposed the application of minimal invasive techniques and the subsequent minimization of the abdominal incisions, efficient anesthesia and perioperative analgesia schemes, optimization of fluid and electrolyte administration and the early onset of mobilization and oral nutrition $(2,3)$.

Adequate management of postoperative pain in colorectal surgery is of utmost importance, since increased, incision related discomfort prevents patients from mobilizing, increase morbidity and deteriorates the overall satisfaction rates $(4,7)$. Multimodal analgesia aims primarily in the deescalation of opioid consumption, due to their severe postoperative side effects (4). In open colectomies, this was translated to the application of thoracic epidural analgesia (8). Nonetheless, in laparoscopic colectomies the optimal analgesia strategy is still not clarified (9).

The intravenous administration of a local anesthetic agent has been also proposed as an alternative method of optimizing postoperative rehabilitation (10). More specifically, the use of lidocaine, a low cost, easy to administer and generally available drug, has yielded promising results (10). Lidocaine provides its analgesic effect through the interaction with several receptors and ion channels and the interference with the nociceptive transmission pathways $(11,12)$. In addition to the analgesic and anti-hyperalgesic properties in acute and neuropathic pain syndromes, by modifying the secretion of specific cytokines, lidocaine decreases the systemic inflammatory response $(11,12)$. Furthermore, the appeal of lidocaine as an adjunct in the ERAS protocols has been reinforced by the validation of its safety profile through various animal and clinical studies (11-15).

The impact of intravenous lidocaine on postoperative recovery has been extensively evaluated (16-18). Although strong evidence regarding its efficacy is still limited, due to the low methodological quality of the current studies, introduction of lidocaine in the perioperative analgesia protocol has been correlated with a reduction in the visual 
analogue scale scores of pain and the cumulative opioid consumption, rendering it an effective adjuvant in abdominal surgery (19). Moreover, the positive impact of lidocaine in postoperative recovery has been separately validated in the open colorectal and the minimal invasive settings $(16,19-21)$.

However, current literature reports regarding the effect of lidocaine on laparoscopic colectomies provide conflicting results $(22,23)$. In the randomized controlled trial by Kaba et al. (24), intravenous infusion of lidocaine accelerated postoperative bowel function and hospital discharge and lowered the requirements for opioid analgesics. Lidocaine has been also associated with a reduction in pain levels and postoperative nausea and vomiting rates $(25,26)$. Subsequent studies $(27,28)$ though, failed to confirm these findings, questioning thus the value of intravenous lidocaine in laparoscopic colectomies. In addition, a meta-analysis of Cooke et al. (29) has shown that intravenous (IV) use of lidocaine reduces time to first bowel movement, postoperative ileus, pain scores, and length of stay in patients that underwent colectomy. Similar were the results of a recent meta-analysis that included only laparoscopic colectomies for postoperative pain, opioid consumption, and length of hospital stay (LOS), but overall postoperative complications were not included as endpoints and the data of two of the included studies were not retrievable (30).

Taking into consideration the above-mentioned evidence, we designed and conducted the present systematic review and meta- analysis of the literature, to provide a current appraisal of the impact of lidocaine administration in the rehabilitation of patients submitted to laparoscopic colectomies for benign or malignant pathologies.

\section{Materials and Methods}

Study protocol. The present study was designed and conducted based on the PRISMA guidelines (31) and the Cochrane Handbook for Systematic Reviews of Interventions.

Primary endpoint. The primary endpoint of this meta-analysis was the validation of difference in the LOS, between perioperative administration of IV lidocaine and saline infusion, in patients who were submitted to laparoscopic colectomy for benign or malignant pathologies.

Secondary endpoints. The following secondary endpoints were also included in the present study: perioperative comparisons (operation duration and morphine consumption), postoperative bowel function (time to first flatus, time to first bowel opened and time to tolerate diet) and total and specific complication rates [postoperative nausea and vomiting - (PONV), urinary retention and urinary tract infections - (UTI), surgical site infection - (SSI), anastomotic leak, ileus and lidocaine toxicity].

Eligibility criteria. Eligible studies were all human randomized controlled trials comparing intravenous lidocaine administration to placebo infusion in laparoscopic colorectal surgery for benign or malignant pathologies, whose outcomes could be retrieved and were reported in English.

Exclusion criteria for the present meta-analysis were: 1) nonhuman studies, 2) outcomes of no interest, 3) not reported in English, 4) irretrievable outcome data, 5) no comparison group, 6) studies in the form of letters, editorials, expert opinion, or conference abstracts.

Literature search. A systematic literature search in the electronic scholar databases (Medline, Scopus, Web of Science, CENTRAL) was performed. The last search date was 01/05/2021. The following MESH terms were introduced in the search algorithm: 'lidocaine', 'lignocaine', 'analgesia', 'laparoscopic', 'minimal', 'laparoscopy', 'colectomy', 'colorectal', 'colon', 'abdominal', 'resection', 'ambulatory'.

Study selection and data collection. After the removal of the duplicate entries, the titles and abstracts of the articles were submitted to a systematic screening, on the basis of the inclusion criteria. After this step, the remaining publications underwent a full text review. Only data provided in the respective publication were extracted. All electronic database screening, study selection, data extraction and quality assessment were performed in duplicate and blindly by two independent researchers (C.S. and K.P.). In case of a discrepancy the two researchers reached a consensus through mutual revision and discussion. If the disagreement was not resolved, the opinion of a third researcher (I.B.) was considered.

From the included studies the following data were extracted: trial characteristics [first author, country, year of completion, sample size, gender, age and body mass index (BMI), follow-up period] patient characteristics [American Society of Anesthesiologists-(ASA) score, underlying pathology, and malignancy stage], operative characteristics (operation type, surgical experience, pneumoperitoneum settings) and anesthesia and analgesia protocol (lidocaine infusion rate, intraoperative analgesia, anesthesia and muscle relaxant medications and postoperative analgesia protocol).

All eligible studies were submitted to rigorous quality and methodological assessment on the basis of the Cochrane's risk of bias assessing tool (32). Through this evaluation tool, the eligible randomized controlled trials (RCTs) were rated in categories such as selection, performance, detection, attrition and reporting bias in the study methodology. Cohen's k statistic was also calculated.

Statistical analysis. Data analysis was performed through the Cochrane Collaboration RevMan version 5.3 and IBM SPSS version 23. The continuous and dichotomous variables were provided in the form of weighted mean difference (WMD) and odds ratio (OR), respectively. The corresponding $95 \%$ confidence interval (95\% CI) was reported for all analysis's estimations.

Considering the continuous variables, if the article did not provide the respective mean (M) and standard deviation (SD), then they were estimated from the reported median, range or interquartile range (IQR), based on the formula of Hozo et al. (33). The MantelHaenszel (MH) and the inverse variance (IV) statistical methods were introduced for the calculations of dichotomous and continuous variables, respectively. Both fixed effects (FE) and random effects (RE) models were estimated. The model that was finally reported in our study was determined by the results of the Cochran Q test. If a statistically significant level of heterogeneity was identified (Q 
test $p<0.1$ ), then the RE model was provided. Heterogeneity was also quantified through the estimation of $\mathrm{I}^{2}$. Statistical significance was considered at the level of $p<0.05$.

Risk of bias across studies. To assess the possible presence of publication bias the funnel plot of the primary endpoint was visually inspected. Moreover, an Egger's test was performed for the primary outcome.

\section{Results}

Study selection. Overall, the application of the search algorithm resulted in the retrieval of 7,503 articles (Figure 1). After the removal of 3,628 duplicate entries, 3,875 titles and abstracts were screened. In total, 3,836 citations (21 conference abstracts editorials or letters, 24 non-human studies, 256 reviews or meta-analyses and 3,535 irrelevant records) were considered as ineligible in this step. The full text review of the remaining 39 articles, resulted in the exclusion of 31 publications ( 5 studies with inadequate data and 25 irrelevant records). Therefore, 8 studies (24-28, 3436) were included in the qualitative and quantitative synthesis of the present study.

Study characteristics. The characteristics of the included studies are summarized in Table I. Overall, 407 patients were introduced in the present meta-analysis. All incorporated trials had an RCT design and the publication date ranged from 2007 (24) to $2018(27,28,35)$. In the majority of the studies(24, 27, 28, 34-36), colectomies were performed laparoscopically, while hand assisted operations were reported only by two studies $(25,26)$. Gender, age and BMI are also reported in Table I. Follow-up period reports were inconsistent.

In total, 154 ASA I, 203 ASA II and 50 ASA III patients were submitted to laparoscopic colectomies. The minority of the included trials provided information regarding the underlying pathology $(24,27,36)$ or the stage of malignant disease (26).

The patient allotment according to the resection type was not associated with major discrepancies. Experience in the minimal invasive techniques was confirmed in four studies (24-27). Only two studies reported the intraoperative pneumoperitoneum pressure settings $(27,34)$.

Lidocaine infusion rates ranged from $1.5 \mathrm{mg} / \mathrm{kg} / \mathrm{h}$ to 2 $\mathrm{mg} / \mathrm{kg} / \mathrm{h}$. Intraoperative analgesia was achieved through administration of fentanyl or other derivatives. For anesthesia maintenance, desoflurane (34), isoflurane (36) or sevoflurane $(24-26,35)$ was provided. Muscle relaxants included agents like rocuronium (25-27), atracurium (28) and cisatracurium $(35,36)$. In most studies opioids were administered postoperatively systemically through a PCA device $(24,27,28,34-36)$. Heterogeneity regarding the remaining analgesic regimen was identified.

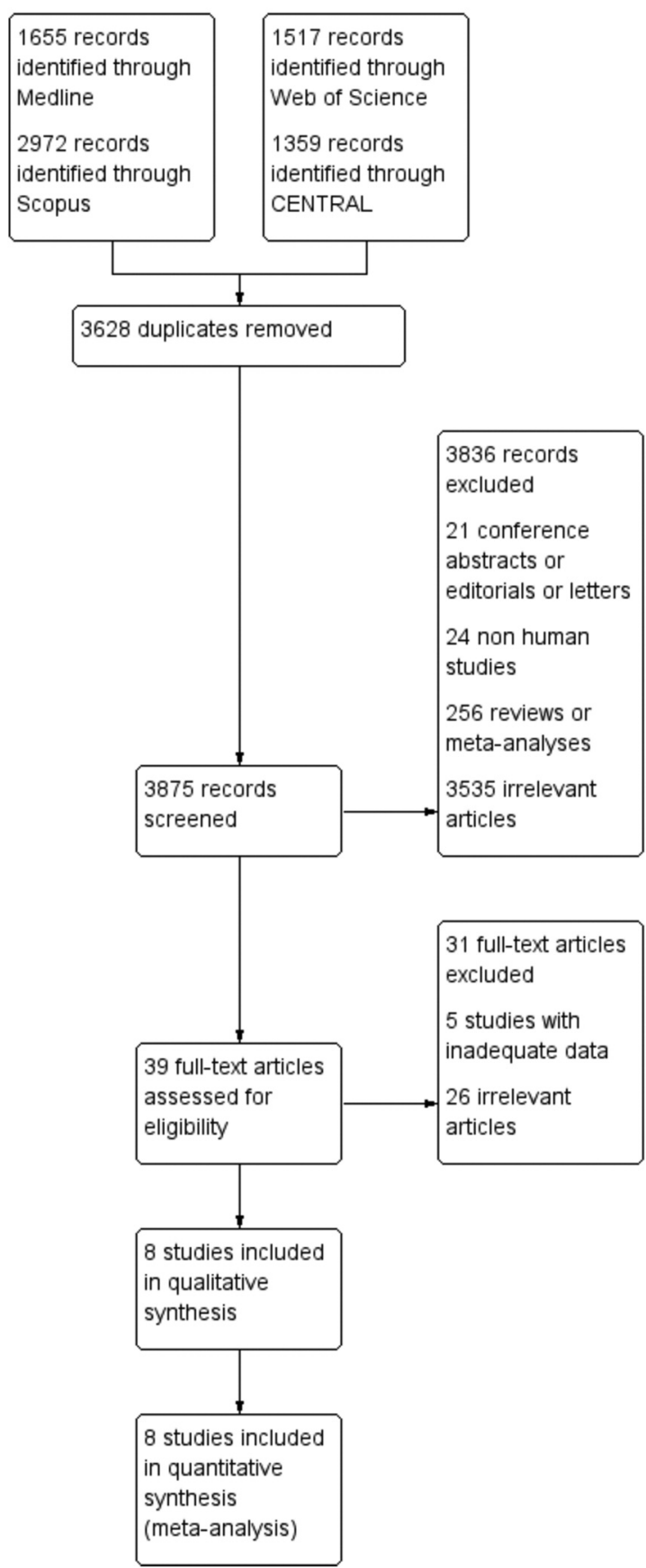

Figure 1. Study flow diagram.

Primary endpoint. Overall, data regarding the effect of perioperative IV lidocaine administration were extracted from 7 studies $(24-28,34,35)$. Meta-analysis of these data (Figure 2) did not display a significant impact $(p=0.23)$ of lidocaine on LOS (WMD: -0.23 , 95\% CI=0.6, 0.14). Heterogeneity was significantly high and therefore an RE (Q 
test $\left.p<0.0001, \mathrm{I}^{2}: 84 \%\right)$ model was applied. Power calculation showed that due to the high levels of heterogeneity, the small average number of participants per group and effect size, our primary endpoint estimations did not achieve adequate statistical power $(17 \%)$.

Due to the high level of heterogeneity, sub-analyses were performed. Sensitivity analysis, revealed that the exclusion of the study of Kim et al. (26), resulted in a significantly lower LOS (WMD: $-0.39, p=0.03$ ) in favor of the lidocaine group, without, though, affecting the heterogeneity levels ( $\mathrm{Q}$ test $p<0.0001, \mathrm{I}^{2}: 81 \%$ ). Metaregression for the variables of age $(p=0.6)$, sample size $(p=0.24)$ and BMI $(p=0.8)$ did not identify a correlating factor. Subgroup analysis after the exclusion of the hand assisted operations did not alter the heterogeneity levels $\left(\mathrm{I}^{2}: 80 \%\right)$ or the pooled results $(p=0.12)$. Further analyses were not implemented, due to the inconsistency of the reported data.

Secondary endpoints. Perioperative comparisons between the lidocaine and placebo group included operation duration and morphine consumption (Figure 3 ). There was no difference between the two study arms in terms of operation duration (WMD: 4.19, 95\%CI=-6.26-14.65, $p=0.43$ ). Morphine consumption was the only perioperative pain assessment related endpoint for which data were retrievable and comparable. Only two trials $(28,36)$ reported on this outcome, and no effect of lidocaine in morphine consumption was identified (WMD: $0.38,95 \% \mathrm{CI}=-15.26$ 16.01, $p=0.96)$. In both outcomes, heterogeneity was significant ( $\mathrm{I}^{2}: 76 \%$ and $\mathrm{I}^{2}: 89$, respectively).

Similarly, postoperative bowel function was not affected by the introduction of IV lidocaine in the analgesic medication scheme. More specifically, time to first flatus (WMD: $-0.13,95 \% \mathrm{CI}=-0.42-0.17)$, time to first bowel opened (WMD: $-0.41,95 \% \mathrm{CI}=-0.94-0.12$ ) and time to tolerate diet (WMD: $-0.10,95 \% \mathrm{CI}=-0.24-0.04$ ) were equivalent between the two groups.

Furthermore, lidocaine did not alter the overall complication rate of laparoscopic colectomies $(\mathrm{OR}=0.72$, $95 \% \mathrm{CI}=0.33-1.59)$. Pooled comparisons showed similar rates of postoperative nausea and vomiting (PONV) $(\mathrm{OR}=0.72,95 \% \mathrm{CI}=0.33-1.59)$. In addition to this, further postoperative complications, such as urinary retention and urinary tract infection (UTI) $(p=1)$, surgical site infection (SSI) $(p=0.54)$, postoperative leak $(p=0.57)$ and ileus $(p=0.32)$ were also comparable between the two study arms. Finally, administration of IV lidocaine did not significantly increase the rate of lidocaine related toxicity events $(\mathrm{OR}=2.08,95 \% \mathrm{CI}=0.33-13.20)$.

Risk of bias within studies. Regarding the methodological and quality evaluation of the included trials. incomplete 


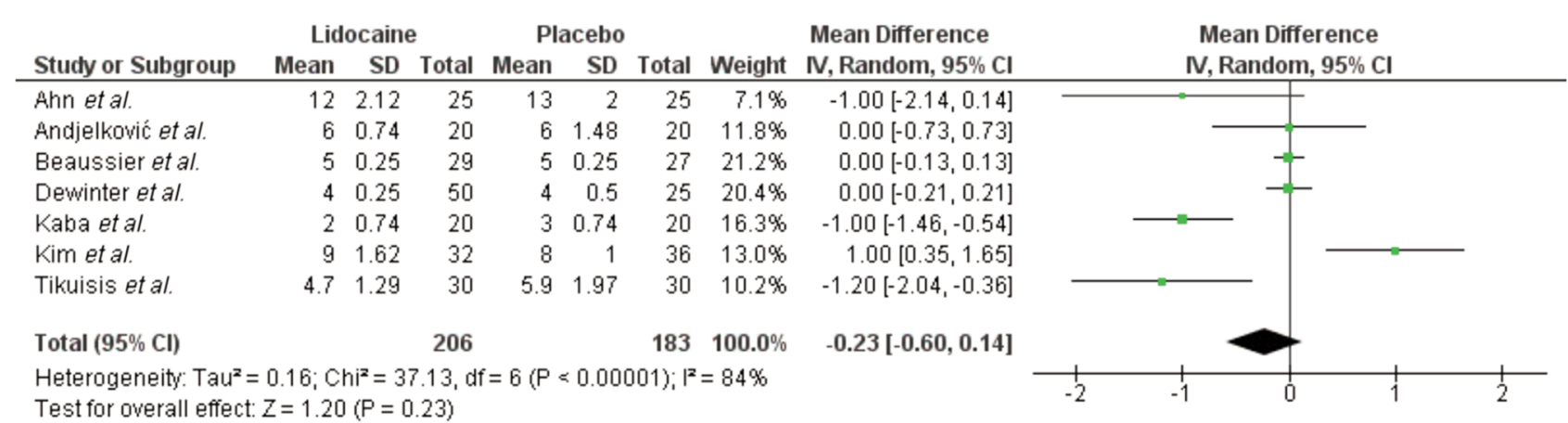

Figure 2. Length of hospital stay.

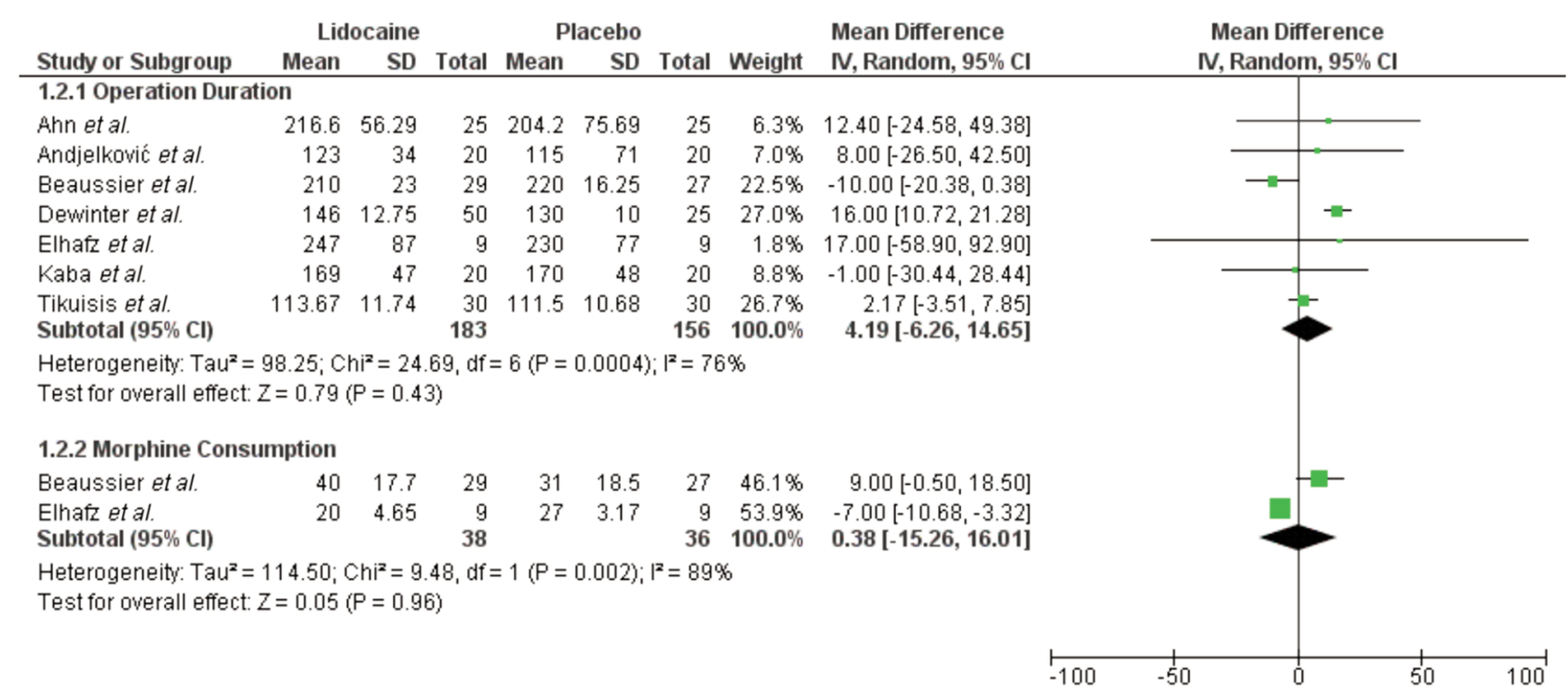

Figure 3. Perioperative comparisons.

outcome data and selective reporting bias was minimal in all studies; however four studies $(28,34-36)$ did not confirm the blinding of the outcome assessor. Moreover, in several studies, the random sequence generation $(28,36)$, the allocation concealment $(28,35,36)$ or the blinding of participants and personnel $(28,36)$ could not be verified in their research protocol. A satisfactory level of interrater agreement was identified (Cohen's k statistic: 92.3\%, $p<0.001)$.

Risk of bias across studies. Visual inspection of the funnel plot of the primary endpoint showed that all included studies were symmetrically distributed and resided within the $95 \%$ CI limits. The calculation of the Egger's test did not confirm the presence of a statistically significant publication bias $(p=0.414)$.

\section{Discussion}

Summary of evidence. The introduction of the ERAS protocols in abdominal operations aimed primarily at the minimization of postoperative morbidity, the acceleration of patient recovery and the subsequent decrease of the hospitalization period (1, 3-5). Besides the obvious effect on the physical and psychological health status of the patients, successful implementation of the ERPs has a major socioeconomic impact $(37,38)$. In order to accomplish a remarkable reduction in the LOS endpoint, several key factors, such as surgical technique, analgesia and anesthesia method, perioperative medication and rehabilitation, should be identified and optimized separately $(3,5,7)$. In the field of laparoscopic colectomy, a pioneering study by Kaba et al. (24), suggested a reduction of the mean hospitalization period by one day, when intravenous lidocaine was applied. Further 
randomized trials though could not support a positive effect of lidocaine on $\operatorname{LOS}(25,26)$. Moreover, meta-analyses provided indeterminate results, regarding the pooled impact of lidocaine on $\operatorname{LOS}(19,20,39)$. Our analysis did not validate a reduction in the hospitalization period when lidocaine was infused. However, our estimations suffered from significant heterogeneity and therefore, the impact of the conclusions is weakened.

Efficient analgesia is a core element of an enhanced recovery protocol. The main lidocaine analgesic pathways, include inhibition of sodium, calcium and potassium channels and the blockade of N-methyl-d-aspartate and G-proteincoupled channels, and the subsequent suppression of $\mathrm{A} \delta$ - and C- fibers, resulting thus in the occlusion of the spontaneous transmissions from the damaged nerve fibers to the proximal dorsal root ganglion $(11,12)$. The analgesic value of intravenous lidocaine has been examined in various abdominal operations (40-42). In a recent RCT by Ho et al. (17) examining patients submitted to open colectomy, continuous 48-hour infusion of low dose lidocaine lowered opioid consumption with no effect on the postoperative pain scores. In laparoscopic colectomy, comparison of lidocaine and placebo in a series of studies, showed a supremacy of the first in terms of cumulative opioid intake and visual analogue scale (VAS) scores $(24,25,34)$. Kim et al. (26), though did not identify a significant difference in postoperative pain or opioid consumption after a 24-hour infusion of lidocaine. Similar were the results from the study group of Beaussier et al. (28). Although a previous meta-analysis (19) suggested a significant early analgesic effect of lidocaine, a recent Cochrane review could not confirm that (39). Our findings are in accordance with the respective literature reports. Although postoperative pain comparisons were unavailable, due to the scarcity and inconsistency of the provided data, overall morphine consumption was not affected by the addition of lidocaine.

The anti-inflammatory properties of lidocaine result from the attenuation of neurogenic inflammation and the reduction of proinflammatory cytokines and neutrophils during the acute phase of the inflammatory response (12). More specifically, studies have shown that intravenous lidocaine administration in sepsis models reduced tumor necrosis factor $\alpha$ (TNF- $\alpha$ ), ICAM-1 and chemokines, improved organ dysfunction, inhibited ischemic injury, enhanced the mucosal barrier and gas exchange during reperfusion, and lessened the overall mortality (12). In laparoscopic colectomy, intravenous lidocaine resulted in a significant decrease in the end-tidal concentration of anesthetics required to maintain hemodynamic stability (24). Furthermore, a reduction of the systematic stress response was described by Ahn et al. (34), where significantly lower C-reactive protein (CRP) levels could be identified two days after surgery.

The combination of intraoperative hemodynamic stability, reduced anesthetic and opioid requirements, inhibition of the systemic inflammatory cascade and attenuation of the sympathetic tone, were expected to have a positive impact on the early bowel mobilization $(2,3,11,23,43)$. At the clinical level, few prospective studies have suggested a significant reduction in the time required for bowel facilitation $(24,25)$. Additionally, a systematic review by Cooke et al. (29), incorporated 405 patients submitted to open or minimal invasive colectomy and concluded that bowel resumption was statistically lower when lidocaine was administered. Nonetheless, subsequent trials could not correlate intravenous lidocaine infusion with early feeding tolerance (26). In the present meta-analysis, 5 studies (24$26,35,36)$ were included and lidocaine was not found to influence the time to first flatus, bowel opened or first diet. Moreover, postoperative ileus rates were comparable.

Finally, safe lidocaine administration is another important aspect that should be considered. Intravenous lidocaine overdosing is associated with cardiovascular (e.g. sinus slowing, elongation of QRS, hypotension, dysrhythmias, shock) and central nervous symptomatology (e.g. dizziness, tinnitus, peri-oral numbness) $(12,15,16,18,42,44)$. Timely recognition of lidocaine toxicity is important for proper treatment (44). Our review showed that RCTs reporting on intravenous lidocaine in laparoscopic colectomies, infused the local anesthetic at a low threshold (maximum 2 $\mathrm{mg} / \mathrm{kg} / \mathrm{h}$ ). Furthermore, pooled comparisons did not identify an increased lidocaine toxicity rate, when compared to saline infusion. In addition to this, lidocaine induced mortality events were not identified in our systematic review, thus confirming the safety profile of intravenous lidocaine administration.

Limitations. Prior to the appraisal of the results of this metaanalysis, several limitations should be acknowledged. First, the pooled result of the primary outcome was statistically underpowered reducing thus the validity of our conclusions. This was attributed mainly to the small number of eligible trials and the inadequate study arm size. Moreover, despite the fact that all included studies incorporated a RCT design and had an adequate methodology and quality appraisal score, significant heterogeneity levels were identified in the majority of the comparisons. Sub-analyses for specific characteristics or individual studies failed to identify heterogeneity introducing factors. Besides the nonhomogeneity in the patient allocation regarding the underlying pathology or the operation type, diversity was also identified in the anesthesia and perioperative analgesia protocol. Bias could also be introduced by the incompetent follow up data. In addition to these, inclusion of further comparisons, such as postoperative pain scores, additional analgesic consumption, or patient satisfaction rates was prohibited by the lack of consistency in the reporting of these endpoints. 


\section{Conclusion}

Our study aimed to provide a pooled estimation of the effect of IV lidocaine administration in the postoperative recovery of patients submitted to laparoscopic colectomy. Meta-analysis data showed that lidocaine did not reduce hospitalization duration or perioperative morphine consumption. Furthermore, we did not identify any alteration in the postoperative bowel function and the complication rates when lidocaine was infused. As a result, we conclude that currently there is no evidence that lidocaine improves postoperative outcomes in laparoscopic colectomies. However, due to several study limitations, further RCTs with methodological standardization and adequate sample size are necessary.

\section{Conflicts of Interest}

The Authors declare no potential conflicts of interest in relation to this study.

\section{Authors' Contributions}

Study conception and design: Balolyiannis I; Acquisition of data: Perivoliotis K, Sarakatsianou C, Samara A; Analysis and interpretation of data: Perivoliotis K, Sarakatsianou C, Samara A; Drafting of manuscript: Perivoliotis K; Critical revision: Tzovaras G, Baloyiannis I.

\section{References}

1 Gustafsson UO, Scott MJ, Schwenk W, Demartines N, Roulin D, Francis N, McNaught CE, MacFie J, Liberman AS, Soop M, Hill A, Kennedy RH, Lobo DN, Fearon K, Ljungqvist $\mathrm{O}$ and Enhanced Recovery After Surgery Society: Guidelines for perioperative care in elective colonic surgery: Enhanced Recovery After Surgery $\left(\right.$ ERAS $\left.^{\circledR}\right)$ Society recommendations. Clin Nutr 31(6): 783-800, 2012. PMID: 23099039. DOI: 10.1016/j.clnu.2012.08.013

2 Ljungqvist $\mathrm{O}$, Scott $\mathrm{M}$ and Fearon KC: Enhanced recovery after surgery: a review. JAMA Surg 152(3): 292-298, 2017. PMID: 28097305. DOI: 10.1001/jamasurg.2016.4952

3 Elhassan A, Elhassan I, Elhassan A, Sekar KD, Rubin RE, Urman RD, Cornett EM and Kaye AD: Essential elements for enhanced recovery after intra-abdominal surgery. Curr Pain Headache Rep 23(3): 21, 2019. PMID: 30854614. DOI: 10.1007/s11916-019-0753-5

4 Simpson JC, Bao X and Agarwala A: Pain management in enhanced recovery after surgery (ERAS) protocols. Clin Colon Rectal Surg 32(2): 121-128, 2019. PMID: 30833861. DOI: 10.1055/s-0038-1676477

5 Ban KA, Berian JR and Ko CY: Does implementation of enhanced recovery after surgery (ERAS) protocols in colorectal surgery improve patient outcomes? Clin Colon Rectal Surg 32(2): 109-113, 2019. PMID: 30833859. DOI: 10.1055/s-0038-1676475

6 Parker JM, Feldmann TF and Cologne KG: Advances in laparoscopic colorectal surgery. Surg Clin North Am 97(3): 547-560, 2017. PMID: 28501246. DOI: 10.1016/j.suc. 2017.01 .005
7 Li D and Jensen CC: Patient satisfaction and quality of life with enhanced recovery protocols. Clin Colon Rectal Surg 32(2): 138144, 2019. PMID: 30833864. DOI: 10.1055/s-0038-1676480

8 Radovanović D, Radovanović Z, Škorić-Jokić S, Tatić M, Mandić A and Ivković-Kapicl T: Thoracic epidural versus intravenous patient-controlled analgesia after open colorectal cancer surgery. Acta Clin Croat 56(2): 244-254, 2017. PMID: 29485791. DOI: 10.20471/acc.2017.56.02.07

9 Perivoliotis K, Sarakatsianou C, Georgopoulou S, Tzovaras G and Baloyiannis I: Thoracic epidural analgesia (TEA) versus patient-controlled analgesia (PCA) in laparoscopic colectomy: a systematic review and meta-analysis. Int $\mathrm{J}$ Colorectal Dis 34(1): 27-38, 2019. PMID: 30519843. DOI: 10.1007/s00384018-3207-3

10 MacFater WS, Xia W, Barazanchi A, Su'a B, Svirskis D and Hill AG: Intravenous local anaesthetic compared with intraperitoneal local anaesthetic in abdominal surgery: a systematic review. World J Surg 42(10): 3112-3119, 2018. PMID: 29666908. DOI: 10.1007/s00268-018-4623-9

11 Nicholson G and Hall GM: Effects of anaesthesia on the inflammatory response to injury. Curr Opin Anaesthesiol 24(4): 370-374, 2011. PMID: 21659870. DOI: 10.1097/ACO. $0 \mathrm{~b} 013 \mathrm{e} 328348729 \mathrm{e}$

12 van der Wal SE, van den Heuvel SA, Radema SA, van Berkum BF, Vaneker M, Steegers MA, Scheffer GJ and Vissers KC: The in vitro mechanisms and in vivo efficacy of intravenous lidocaine on the neuroinflammatory response in acute and chronic pain. Eur J Pain 20(5): 655-674, 2016. PMID: 26684648. DOI: 10.1002/ejp.794

13 Lee MW, Or DY, Tsang AC, Ng DC, Chen PP, Cheung MH, Li RS and Leong HT: Intravenous lignocaine infusion facilitates acute rehabilitation after laparoscopic colectomy in the Chinese patients. Hong Kong Med J 23(5): 441-445, 2017. PMID: 28126972. DOI: $10.12809 / \mathrm{hkmj} 164984$

14 Iacob E, Hagn EE, Sindt J, Brogan S, Tadler SC, Kennington KS, Hare BD, Bokat CE, Donaldson GW, Okifuji A and Junkins SR: Tertiary care clinical experience with intravenous lidocaine infusions for the treatment of chronic pain. Pain Med 19(6): 1245-1253, 2018. PMID: 29016948. DOI: 10.1093/pm/pnx167

15 Daykin H: The efficacy and safety of intravenous lidocaine for analgesia in the older adult: a literature review. Br J Pain 11(1): 23-31, 2017. PMID: 28386401. DOI: 10.1177/2049463716676205

16 Kranke P, Jokinen J, Pace NL, Schnabel A, Hollmann MW, Hahnenkamp K, Eberhart LH, Poepping DM and Weibel S: Continuous intravenous perioperative lidocaine infusion for postoperative pain and recovery. Cochrane Database Syst Rev (7): CD009642, 2015. PMID: 26184397. DOI: 10.1002/ 14651858.CD009642.pub2

17 Ho MLJ, Kerr SJ and Stevens J: Intravenous lidocaine infusions for 48 hours in open colorectal surgery: a prospective, randomized, double-blinded, placebo-controlled trial. Korean J Anesthesiol 71(1): 57-65, 2018. PMID: 29441176. DOI: 10.4097/kjae.2018.71.1.57

18 Bazin P, Padley J, Ho M, Stevens J and Ben-Menachem E: The effect of intravenous lidocaine infusion on bispectral index during major abdominal surgery. J Clin Monit Comput 32(3): 533-539, 2018. PMID: 28623471. DOI: 10.1007/s10877-0170035-x

19 Weibel S, Jokinen J, Pace NL, Schnabel A, Hollmann MW, Hahnenkamp K, Eberhart LH, Poepping DM, Afshari A and 
Kranke P: Efficacy and safety of intravenous lidocaine for postoperative analgesia and recovery after surgery: a systematic review with trial sequential analysis. Br J Anaesth 116(6): 770-783, 2016. PMID: 27199310. DOI: 10.1093/bja/ aew 101

20 Ventham NT, Kennedy ED, Brady RR, Paterson HM, Speake D, Foo I and Fearon KC: Efficacy of intravenous lidocaine for postoperative analgesia following laparoscopic surgery: a metaanalysis. World J Surg 39(9): 2220-2234, 2015. PMID: 26044546. DOI: 10.1007/s00268-015-3105-6

21 MacFater WS, Rahiri JL, Lauti M, Su'a B and Hill AG: Intravenous lignocaine in colorectal surgery: a systematic review. ANZ J Surg 87(11): 879-885, 2017. PMID: 28677829. DOI: $10.1111 /$ ans. 14084

22 Joshi GP, Bonnet F, Kehlet H and PROSPECT collaboration: Evidence-based postoperative pain management after laparoscopic colorectal surgery. Colorectal Dis 15(2): 146-155, 2013. PMID: 23350836. DOI: 10.1111/j.1463-1318.2012. 03062.x

23 De Oliveira GS Jr, Fitzgerald P, Streicher LF, Marcus RJ and McCarthy RJ: Systemic lidocaine to improve postoperative quality of recovery after ambulatory laparoscopic surgery. Anesth Analg 115(2): 262-267, 2012. PMID: 22584558. DOI: 10.1213/ANE.0b013e318257a380

24 Kaba A, Laurent SR, Detroz BJ, Sessler DI, Durieux ME, Lamy ML and Joris JL: Intravenous lidocaine infusion facilitates acute rehabilitation after laparoscopic colectomy. Anesthesiology 106(1): 11-8; discussion 5-6, 2007. PMID: 17197840. DOI: 10.1097/00000542-200701000-00007

25 Tikuišis R, Miliauskas P, Samalavičius NE, Žurauskas A, Samalavičius R and Zabulis V: Intravenous lidocaine for postoperative pain relief after hand-assisted laparoscopic colon surgery: a randomized, placebo-controlled clinical trial. Tech Coloproctol 18(4): 373-380, 2014. PMID: 24030782. DOI: 10.1007/s10151-013-1065-0

26 Kim HO, Lee SR, Choi WJ and Kim H: Early oral feeding following laparoscopic colorectal cancer surgery. ANZ J Surg 84(7-8): 539-544, 2014. PMID: 24612414. DOI: 10.1111/ ans. 12550

27 Andjelković L, Novak-Jankovič V, Požar-Lukanovič N, Bosnić $\mathrm{Z}$ and Spindler-Vesel A: Influence of dexmedetomidine and lidocaine on perioperative opioid consumption in laparoscopic intestine resection: a randomized controlled clinical trial. J Int Med Res 46(12): 5143-5154, 2018. PMID: 30209962. DOI: $10.1177 / 0300060518792456$

28 Beaussier M, Parc Y, Guechot J, Cachanado M, Rousseau A, Lescot $\mathrm{T}$ and CATCH Study Investigators: Ropivacaine preperitoneal wound infusion for pain relief and prevention of incisional hyperalgesia after laparoscopic colorectal surgery: a randomized, triple-arm, double-blind controlled evaluation $v s$. intravenous lidocaine infusion, the CATCH study. Colorectal Dis 20(6): 509-519, 2018. PMID: 29352518. DOI: 10.1111/ codi. 14021

29 Cooke C, Kennedy ED, Foo I, Nimmo S, Speake D, Paterson HM and Ventham NT: Meta-analysis of the effect of perioperative intravenous lidocaine on return of gastrointestinal function after colorectal surgery. Tech Coloproctol 23(1): 15-24, 2019. PMID: 30721376. DOI: 10.1007/s10151-019-1927-1

30 Wei S, Yu-Han Z, Wei-Wei J and Hai Y: The effects of intravenous lidocaine on wound pain and gastrointestinal function recovery after laparoscopic colorectal surgery. Int Wound J 17(2): 351-362, 2020. PMID: 31837112. DOI: 10.1111/ iwj.13279

31 Moher D, Liberati A, Tetzlaff J, Altman DG and PRISMA Group: Preferred reporting items for systematic reviews and meta-analyses: the PRISMA statement. PLoS Med 6(7): e1000097, 2009. PMID: 19621072. DOI: 10.1371/journal. pmed.1000097

32 Higgins JP, Altman DG, Gøtzsche PC, Jüni P, Moher D, Oxman AD, Savovic J, Schulz KF, Weeks L, Sterne JA, Cochrane Bias Methods Group and Cochrane Statistical Methods Group: The Cochrane Collaboration's tool for assessing risk of bias in randomised trials. BMJ 343: d5928, 2011. PMID: 22008217. DOI: $10.1136 /$ bmj.d5928

33 Hozo SP, Djulbegovic B and Hozo I: Estimating the mean and variance from the median, range, and the size of a sample. BMC Med Res Methodol 5: 13, 2005. PMID: 15840177. DOI: 10.1186/1471-2288-5-13

34 Ahn E, Kang H, Choi GJ, Park YH, Yang SY, Kim BG and Choi SW: Intravenous lidocaine for effective pain relief after a laparoscopic colectomy: a prospective, randomized, doubleblind, placebo-controlled study. Int Surg 100(3): 394-401, 2015. PMID: 25785316. DOI: 10.9738/INTSURG-D-14-00225.1

35 Dewinter G, Coppens S, Van de Velde M, D'Hoore A, Wolthuis A, Cuypers E and Rex S: Quadratus lumborum block versus perioperative intravenous lidocaine for postoperative pain control in patients undergoing laparoscopic colorectal surgery: a prospective, randomized, double-blind controlled clinical trial. Ann Surg 268(5): 769-775, 2018. PMID: 30004914. DOI: 10.1097/SLA.0000000000002888

36 Elhafz AA, Elgebaly AS, Bassuoni AS and El Dabaa AA: Is lidocaine patch as effective as intravenous lidocaine in pain and illus reduction after laparoscopic colorectal surgery? A randomized clinical trial. Anesth Essays Res 6(2): 140-146, 2012. PMID: 25885606. DOI: 10.4103/0259-1162.108291

37 Thanh NX, Chuck AW, Wasylak T, Lawrence J, Faris P, Ljungqvist $\mathrm{O}$, Nelson $\mathrm{G}$ and Gramlich LM: An economic evaluation of the Enhanced Recovery After Surgery (ERAS) multisite implementation program for colorectal surgery in Alberta. Can J Surg 59(6): 415-421, 2016. PMID: 28445024. DOI: $10.1503 /$ cjs.006716

38 Rinninella E, Persiani R, D’Ugo D, Pennestrì F, Cicchetti A, Di Brino E, Cintoni M, Miggiano GAD, Gasbarrini A and Mele MC: NutriCatt protocol in the Enhanced Recovery After Surgery (ERAS) program for colorectal surgery: The nutritional support improves clinical and cost-effectiveness outcomes. Nutrition 50: 74-81, 2018. PMID: 29547797. DOI: 10.1016/j.nut.2018.01.013

39 Weibel S, Jelting Y, Pace NL, Helf A, Eberhart LH, Hahnenkamp K, Hollmann MW, Poepping DM, Schnabel A and Kranke P: Continuous intravenous perioperative lidocaine infusion for postoperative pain and recovery in adults. Cochrane Database Syst Rev 6: CD009642, 2018. PMID: 29864216. DOI: 10.1002/14651858.CD009642.pub3

40 Maquoi I, Joris JL, Dresse C, Vandenbosch S, Venneman I, Brichant JF and Hans GA: Transversus abdominis plane block or intravenous lignocaine in open prostate surgery: a randomized controlled trial. Acta Anaesthesiol Scand 60(10): 1453-1460, 2016. PMID: 27507582. DOI: 10.1111/aas.12773

41 Samimi S, Taheri A and Davari Tanha F: Comparison between intraperitoneal and intravenous lidocaine for postoperative 
analgesia after elective abdominal hysterectomy, a double-blind placebo controlled study. J Family Reprod Health 9(4): 193-198, 2015. PMID: 27047566.

42 Terkawi AS, Tsang S, Kazemi A, Morton S, Luo R, Sanders DT, Regali LA, Columbano H, Kurtzeborn NY and Durieux ME: A clinical comparison of intravenous and epidural local anesthetic for major abdominal surgery. Reg Anesth Pain Med 41(1): 28-36, 2016. PMID: 26650426. DOI: 10.1097/AAP.0000000000000332

43 Ebert TJ, Mohanty PK and Kampine JP: Lidocaine attenuates efferent sympathetic responses to stress in humans. J Cardiothorac Vasc Anesth 5(5): 437-443, 1991. PMID: 1932648. DOI: 10.1016/1053-0770(91)90116-b
44 Hasan B, Asif T and Hasan M: Lidocaine-induced systemic toxicity: a case report and review of literature. Cureus 9(5): e1275, 2017. PMID: 28652958. DOI: 10.7759/cureus.1275
Received July 12, 2021

Revised August 3, 2021

Accepted August 4, 2021 